\title{
BMJ Open Prostate cancer diagnostic pathway: Is a one-stop cognitive MRI targeted biopsy service a realistic goal in everyday practice? A pilot cohort in a tertiary referral centre in the UK
}

\author{
Edward James Bass, ${ }^{1,2}$ Alex Freeman, ${ }^{3}$ Charles Jameson, ${ }^{3}$ Shonit Punwani, ${ }^{4,5}$ \\ Caroline M Moore, ${ }^{1,2}$ Manit Arya, ${ }^{2}$ Mark Emberton, ${ }^{1,2}$ Hashim Uddin Ahmed ${ }^{6,7}$
}

To cite: Bass EJ, Freeman A, Jameson C, et al. Prostate cancer diagnostic pathway: Is a one-stop cognitive MRI targeted biopsy service a realistic goal in everyday practice? A pilot cohort in a tertiary referral centre in the UK. BMJ Open 2018;8:e024941. doi:10.1136/ bmjopen-2018-024941

\section{- Prepublication history for} this paper is available online. To view these files, please visit the journal online (http://dx.doi. org/10.1136/bmjopen-2018024941).

Received 4 July 2018

Revised 24 August 2018

Accepted 20 September 2018

Check for updates

(C) Author(s) (or their employer(s)) 2018. Re-use permitted under CC BY-NC. No commercial re-use. See rights and permissions. Published by BMJ.

For numbered affiliations see end of article.

Correspondence to Dr Edward James Bass; edward.bass@nhs.net

\section{ABSTRACT}

Objectives To evaluate the feasibility of a novel multiparametric MRI (mpMRI) and cognitive fusion transperineal targeted biopsy (MRTB) led prostate cancer (PCa) diagnostic service with regard to cancer detection and reducing time to diagnosis and treatment.

Design Consecutive men being investigated for possible PCa under the UK 2-week wait guidelines.

Setting Tertiary referral centre for PCa in the UK.

Participants Men referred with a raised prostate-specific antigen (PSA) or abnormal digital rectal examination between February 2015 and March 2016 under the UK 2-week rule guideline.

Interventions An mpMRI was performed prior to patients attending clinic, on the same day. If required, MRTB was offered. Results were available within 48 hours and discussed at a specialist multidisciplinary team meeting. Patients returned for counselling within 7 days

Primary and secondary outcome measures Outcome measures in this regard included the time to diagnosis and treatment of patients referred with a suspicion of PCa. Quality control outcome measures included clinically significant and total cancer detection rates.

Results 112 men were referred to the service. 111 (99.1\%) underwent mpMRI. Median PSA was $9.4 \mathrm{ng} / \mathrm{mL}$ (IQR 5.6-21.0). 87 patients had a target on mpMRI with 25 scoring Likert 3/5 for likelihood of disease, $264 / 5$ and 36 5/5. 57 (51\%) patients received a local anaesthetic, Magnetic resonance imaging targeted biopsy (MRTB). Cancer was detected in 45 (79\%). 43 (96\%) had University College London definition 2 disease or greater. The times to diagnosis and treatment were a median of 8 and 20 days, respectively.

Conclusions This approach greatly reduces the time to diagnosis and treatment. Detection rates of significant cancer are high. Similar services may be valuable to patients with a potential diagnosis of $\mathrm{PCa}$.

\section{INTRODUCTION}

Accurate risk stratification for men presenting with localised prostate cancer is vitally important. In its absence, patient-centred

\section{Strengths and limitations of this study}

- First prospective study demonstrating the clinical feasibility of a 'one stop', rapid diagnostic prostate cancer pathway, using both multiparametric MRI (mpMRI) and transperineal targeted biopsy.

- Inclusion criteria reflecting 'real world' practice in the UK.

- This study incorporates a standardised mpMRI acquisition and a validated system for defining clinically significant prostate cancer.

- Cognitive targeted biopsy performed only, rather than $\mathrm{mpMRI} / \mathrm{ultrasound}$ fusion.

- Transperineal, rather than transrectal approach offers minimal septic complications postbiopsy.

management cannot be offered. Men with low-risk disease can be safely managed with active surveillance, whereas men with a good life expectancy and intermediate to highrisk disease are likely to benefit from interventional treatment. ${ }^{1}$ Currently, standard practice uses prostate-specific antigen (PSA) value, digital rectal examination (DRE) and transrectal ultrasound guided biopsy (TRUSGB). However, TRUSGB is inherently random. The tumour cannot be visualised with certainty, and thus leads to overdiagnosis of insignificant disease in up to $50 \%$ of men, ${ }^{3}$ and missing significant disease in $18 \%$ of men, especially if cancer is located in the anterior or apical regions of the prostate. ${ }^{4}$ This creates difficulty for urologists and adds anxiety to patients ${ }^{5}$ who have to undergo a repetitive cascade of diagnostic tests which inevitably has cost implications for healthcare providers.

Transperineal mapping (TPM) or zonal biopsies of the prostate offer a diagnostic alternative to TRUSGB with demonstrable 
diagnostic success. However, the burden on patients is high. First, the extensive biopsies demand general anaesthesia. Second, the rates of urinary retention following the procedure are high, making postoperative catheterisation commonplace. Third, the large number of cores taken requires many hours of labour to assess. Thus, a patient may have to wait significantly longer for a result, adding to their anxiety. This may also delay necessary treatment. Whether this results in adverse outcomes is not known. However, all of these established difficulties do confer added costs. Indeed, if every patient undergoing TRUSGB instead underwent a TPM, the cost of such a move would likely be exceedingly high. Therefore, the challenge presents itself as biopsy offering superior clinically significant detection rates to the existing standard, while not conferring an added cost.

Multiparametric MRI (mpMRI) of the prostate has proved a useful tool in the diagnosis and risk stratification of prostate cancer. MpMRI has demonstrated its ability to detect significant cancers, while not detecting those which are insignificant. ${ }^{4}$ Suspicious areas on mpMRI can be targeted with subsequent transperineal biopsy (MRTB). MRTB has demonstrated greater sampling efficiency and accuracy when compared with standard TRUSGB proto$\operatorname{cols}^{6-8}$ and has demonstrated accuracy when compared with the reference standard of radical prostatectomy (RP).$^{9}$ This allows for a more accurate assessment of Gleason grade, and therefore an improved risk stratification and treatment plan at diagnosis. ${ }^{10}$ Furthermore, the efficiency advantage, that is, taking fewer cores at biopsy, confers significant benefits in cost, patient tolerability and postbiopsy sepsis rates.

Three methods of transperineal MRTB currently exist. First and most common is 'cognitive targeting'. This approach requires the urologist to review the mpMRI images and aim the needle towards the corresponding area on ultrasound imaging. ${ }^{11}$ Alternatively, the reporting uroradiologist draws a diagrammatic representation of the gland and any suspicious area contained within, which guides the urologist to potential cancer. Second, 'in-bore MRTB' is performed while the patient is in the MRI scanner, allowing for real-time targeting of suspicious areas with MRI-compatible biopsy equipment. Third, 'fusion targeting' uses specifically designed software to allow combination of the mpMRI images with real-time ultrasound imaging. ${ }^{4}$ The latter two methods have implications in terms of equipment availability and cost, and as of yet, the question of superiority of any one over another remains elusive. ${ }^{4}$

Currently, prostate cancer diagnostic pathways remain built around TRUSGB. MPMRI is more commonly being used prior to TRUSGB. However, the use of an mpMRI and MRTB pathway remains a rarity despite the potential advantages of such an approach and the novel approach of both diagnostic interventions in 1 day exceptionally so. The reasons for this are multiple and commonly relate to the techniques being in their relative infancy. The lack of standardised mpMRI reporting, ${ }^{12}$ a learning curve for operators, ${ }^{13}$ mpMRI availability and $\operatorname{cost}^{14}$ and concern regarding missed diagnosis from not sampling the whole gland have all been cited as reasons not to accept widespread adoption. Despite this, MRI-guided targeted biopsy pathways have been used before, although via the transrectal rather than the transperineal route. ${ }^{15-17}$ The recent findings of the PRostate Evaluation for Clinically Important disease: Sampling using Image-guidance Or Not? (PRECISION) ${ }^{18}$ trial has clearly addressed concerns with regard to superiority of an MRI-targeted biopsy approach over systematic TRUS biopsy, demonstrating superiority in clinically significant cancer detection rate and a reduction in the detection of insignificant disease.

Thus, the objective of this pilot study was primarily to determine the suitability and feasibility of a 'One-Stop', transperineal MRI-targeted biopsy pathway for prostate cancer in 'real-world' clinical practice. Outcome measures in this regard included the time to diagnosis and treatment of patients referred with a suspicion of prostate cancer. Quality control outcome measures included clinically significant and total cancer detection rates.

\section{PATIENTS AND METHODS}

This prospective study analyses the clinical and service outcomes of an mpMRI and MRTB-led prostate cancer diagnostic pathway (figure 1) from February 2015 to March 2016. Inclusion criteria were men presenting with a biochemical or clinical suspicion of prostate cancer under the UK 2-week wait programme and undergoing mpMRI and if necessary subsequent cognitive targeted prostate biopsy. Patients without negative urine cultures or with estimated glomerular filtration rates of $<30 \mu \mathrm{mol} / \mathrm{L}$ were excluded. The patient was contacted on referral, and an mpMRI was arranged. This was reported before the patient attended clinic in the early afternoon of the same day. If a targetable lesion was identified (Likert $\geq 4$ ), a transperineal-targeted biopsy was advised. If a target was rated as equivocal (Likert=3), the discussion was more nuanced including risk factors for a subsequent biopsy being positive such as a positive family history of prostate cancer, high PSA density or concordant positive DRE findings. Further, in this group of men, those with diffuse equivocal changes requiring a greater number of cores to be taken for a positive result, the option of full template biopsies under general anaesthetic was discussed. Results were available within 48 hours and were discussed at a specialist multidisciplinary team. Patients returned for counselling within 7 days.

MpMRI acquisition was performed according to the European guidelines of uroradiology previously described by the University College London (UCL) group. ${ }^{12} 19{ }^{20}$ In summary, this includes the use of a 1.5 or 3.0 Tesla MRI scanner acquiring T2-weighted axial and coronal, axial diffusion weighted coefficient (DWI) and high b-value, as well as T1-weighted dynamic contrast enhancement (intravenous Gadolinium) images (DCE). Each scan was reported by an experienced uroradiologist as previously 

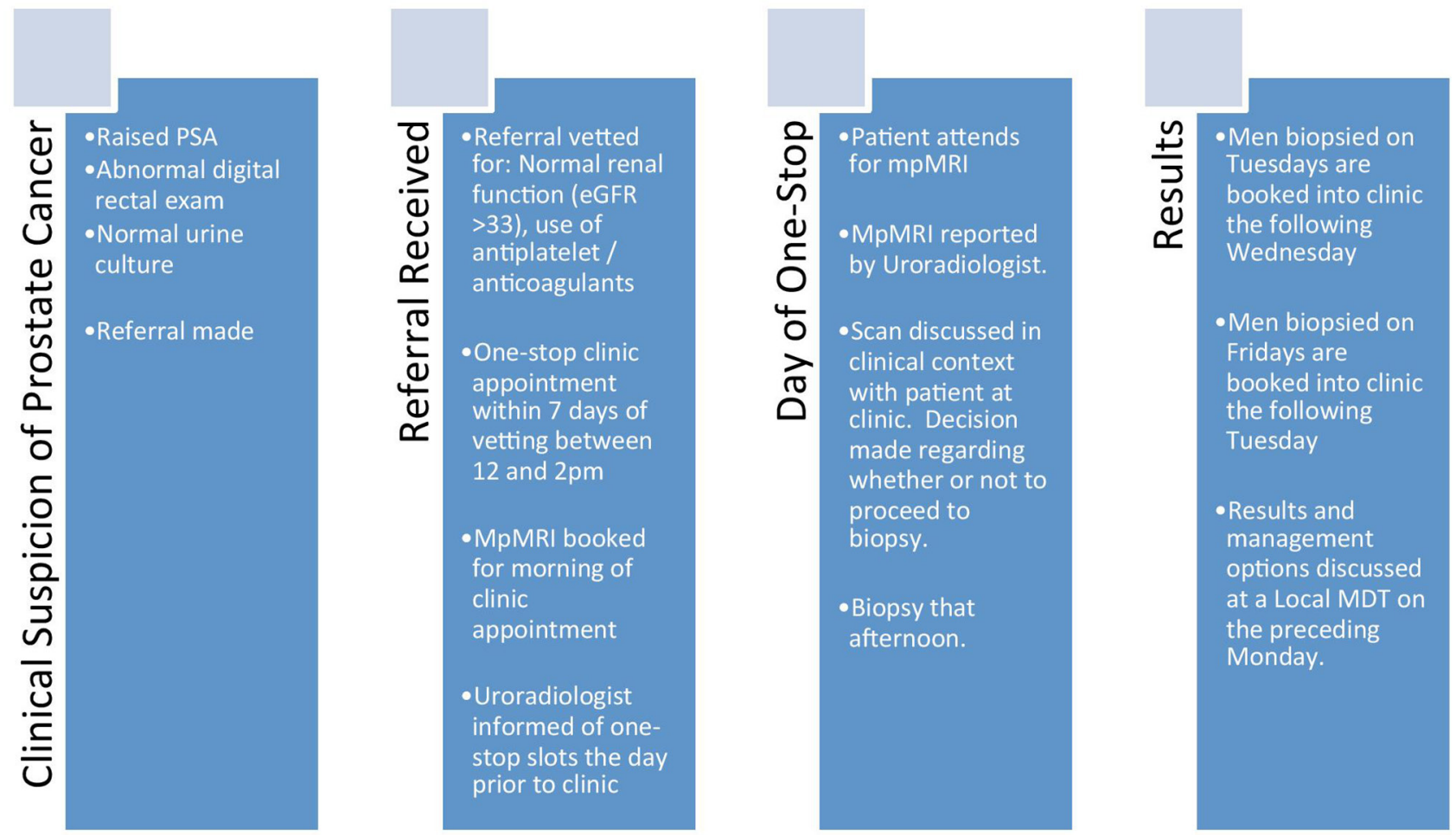

Figure 1 The One-Stop mpMRI-led MRTB prostate cancer diagnostic pathway. MDT, multidisciplinary team; mpMRI, multiparametric MRI; PSA, prostate-specific antigen.

described $^{21} 22$ and a pictorial diagrammatic map drawn (figure 2). Regions of interest (ROIs) were scored using a Likert-like scale of $1-5^{22}$ using the overall impression of the radiologist to characterise the level of suspicion for prostate cancer. ROIs scoring 4 or 5 were thought 'likely' or 'highly likely' to contain a malignant lesion which was either $\geq 0.2 \mathrm{~mL}$ in volume and/or had high-grade components within (Gleason $\geq 3+4$ ) ${ }^{23}$ ROIs 3 were rated as indeterminate for such disease and this score of 3 , or higher, was chosen as the threshold for a positive mpMRI. Our choice of scoring system was based on the outcomes of the 2011 European Consensus Meeting ${ }^{12}$ which met prior to the Prostate Imaging and Data Reporting System (PIRADS) mpMRI reporting consensus meeting ${ }^{19}$ and has demonstrated equivalency with the PIRADS system. ${ }^{24}$

The procedure was performed as a day case under local anaesthesia and antimicrobial prophylaxis in the lithotomy position, by either a consultant urologist or urology clinical fellow as previously described. ${ }^{25}$ This biopsy technique has demonstrated a median procedure length of $30 \mathrm{~min}$ and good patient toleration, with median visual analogue pain scores of 1.0. ${ }^{26}$

Data were collected on a case report form compliant with the Standards of Reporting for MRI-targeted Biopsy Studies of the prostate. ${ }^{11}$ Included data were patients demographics, indications for biopsy, PSA value, prostate volume, number of targets per patient and Likert score per target. ${ }^{11}$ Additionally, for each biopsy collected the total number of cores taken, biopsy density, number of positive cores, maximum and overall Gleason scores and the maximum cancer core length (MCCL). Biopsy efficiency was calculated by the number of cores demonstrating clinically significant disease divided by the number of cores taken. For the purpose of this study, clinically significant disease was defined using the UCL classification for interpreting transperineal biopsy findings which sets the significance threshold at Gleason score $>/=$ to $3+4$ and/ or MCCL $\geq 4 \mathrm{~mm}$ for definition 2 and $>/=$ to $4+3$ and/or MCCL $\geq 6 \mathrm{~mm}$ for definition $1^{26}$ (figure 3 ).

Finally, to assess the time to diagnosis and treatment as well as the treatments elected by men were determined by examination of the hospital trust's electronic data system.

\section{Patient and public involvement}

Participants were not involved in the design of the study. However, conclusions gleaned from the study are to be disseminated among patients newly referred to the service.

\section{RESULTS}

\section{Patient demographics}

In total, 112 consecutive biopsy naive men with a median age of 68 attended the prostate cancer one stop clinic between February 2015 and March 2016 (table 1A). All but one man $(99 \%)$ received an mpMRI scan prior to clinic. The patient in question had an MRI incompatible cardiac pacemaker.

\section{MpMRI outcomes}

The median prostate volume was $50 \mathrm{~mL}$. Eighty-seven men $(78 \%)$ had a positive mpMRI (Likert score $\geq 3$ ) and 24 (22\%) had a negative scan (Likert score $\leq 2$ ) and did not go on to biopsy. Twenty-five men (29\%) had an mpMRI scan 
SVs

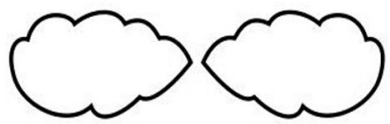

Base

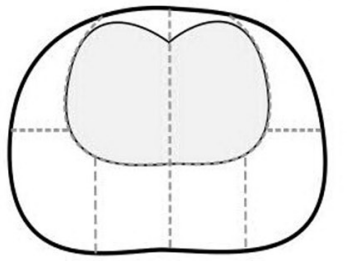

Midgland

Apex
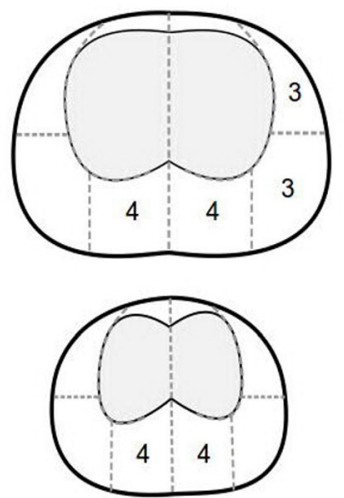

(O)
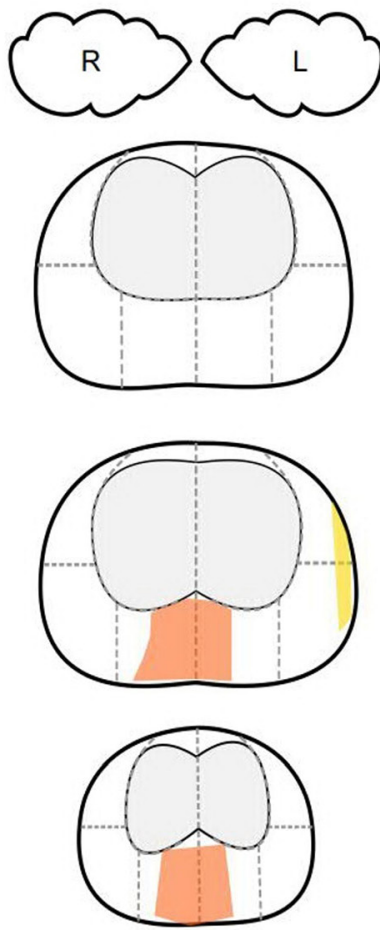

(O)

Figure 2 A pictorial prostate mpMRI diagrammatic report, as drawn by the uroradiologist. mpMRI, multiparametric MRI; SV, Seminal vesicle; R, Right; L, Left

with an overall Likert score of 3, $26(30 \%)$ an overall score of 4 and $36(41 \%)$ an overall Likert score of 5 . There were 162 ROIs identified on mpMRI with a median volume of $0.5 \mathrm{~mL}$ when measured on T2 MRI sequencing. Thirty-nine men (45\%) had a single ROI on mpMRI, 25 men (29\%) had two, 22 men (25\%) had three and a single man (1\%) had four. Seventy-one lesions (30\%) were Likert 3, 49 (30\%) were Likert 4 and 42 (26\%) were Likert 5 . After mpMRI, nine with negative mpMRIs (38\%) were discharged for PSA surveillance in the community, $10(42 \%)$ remained on PSA surveillance in secondary care, four (17\%) underwent investigations for lower urinary tract symptoms and one $(4 \%)$ underwent a full template biopsy under general anaesthetic (table 1B).

\section{Biopsy outcomes}

Fifty-seven men (51\%) underwent a local anaesthetic MRTB as described following mpMRI (table 1C). Fifteen (17\%) men chose not to undergo biopsy under local anaesthetic and were listed for a biopsy under sedation. Thirteen men (15\%) did not have a biopsy due to clinical reasons. Any cancer was detected in $45(79 \%)$ men. Of these, 43 (96\%) satisfied the UCL 2 criteria for clinical significance and 34 $(76 \%)$ satisfying the UCL 1 criteria. The median MCCL of positive biopsies was $7 \mathrm{~mm}$. The calculated biopsy efficiency for UCL 2 disease was 47\%. The median number of cores taken per ROI was 4 , with a median calculated biopsy density of 10 cores $/ \mathrm{mL}$ of ROI. Of the 20 men who had more than one lesion on mpMRI and underwent biopsy, two had a secondary lesion which harboured either higher grade or volume disease. In only one of these men was the secondary lesion a lower Likert score. Both such men went on to RP.

\section{Diagnosis and treatment outcomes}

The median time to a man being told his diagnosis was 8 days, and the median time by which treatment had been started was 20 days, although in five cases this time period was not clear (table 1D). The treatment outcomes are shown in table 1D. Of note, $20(18 \%)$ men were discharged after biopsy with 19 (17\%) men starting PSA surveillance. Forty-four (40\%) went on to undergo treatment, and nine $(8 \%)$ men underwent a further biopsy either due to a perceived false negative or diffuse disease requiring a biopsy under sedation or general anaesthetic. Eleven (10\%) patients underwent further assessment or treatment for benign disease.

\section{DISCUSSION}

An optimal prostate cancer diagnostic strategy should encapsulate maximal significant cancer detection while avoiding insignificant disease or repeat biopsy. Furthermore, it should convey enough information for urologists and patients to accurately devise a treatment plan according to the risk of 
Table 1 Continued

\begin{tabular}{lll}
\hline \multicolumn{4}{l}{ D. Diagnosis and treatment outcomes } \\
\hline Median time to diagnosis (days) & \multicolumn{2}{l}{8 (IQR 5-12) } \\
Median time to treatment (days) & \multicolumn{2}{l}{20 (IQR 8-40) } \\
\hline Treatment type (postbiopsy) & $\mathrm{n}$ & $\%$ \\
\hline Discharged & 4 & 7 \\
\hline PSA surveillance & 6 & 11 \\
Active surveillance & 5 & 9 \\
\hline Focal therapy & 6 & 11 \\
Robotic prostatectomy & 9 & 16 \\
\hline External beam radiotherapy & 10 & 18 \\
\hline Brachytherapy & 2 & 4 \\
Androgen deprivation therapy & 9 & 16 \\
Chemotherapy & 4 & 7 \\
Antibiotics & 1 & 2 \\
Repeat biopsy & 1 & 2 \\
\hline
\end{tabular}

MCCL, maximum cancer core length; mpMRI, multiparametric MRI; PSA, prostate-specific antigen; ROI, regions of interest; UCL, University College London.

concerns regarding its cost, need for general anaesthetic, increased complications and patient burden. Such concerns have justly prevented its wider use and certainly a TPM-led diagnostic pathway has not been seriously suggested.

However, the development and refinement of mpMRI demands that its use in leading an approach to diagnosis must be contemplated. MpMRI has demonstrated high levels of accuracy for the detection of clinically significant cancer when compared with both $\mathrm{TPM}^{37}$ and whole-mount prostatectomy specimens. ${ }^{9}$ Indeed, a systematic review by Fütterer et al found that mpMRI detected clinically significant disease in up to $84 \%$ of men with an NPV of up to $98 \%$ where either TPM or prostatectomy was used as the reference standard. ${ }^{20}$ More recently, the results of the Prostate MR Imaging Study (PROMIS) trial demonstrate the sensitivity and NPV of mpMRI in detecting clinically significant disease as $93 \%$ and $89 \%$, respectively. ${ }^{38}$ Furthermore, the PROMIS trial demonstrated that $27 \%$ of men could avoid a biopsy. ${ }^{38}$ Despite these findings, both the European Association of Urology ${ }^{39}$ and the National Institute for Health and Care Excellence ${ }^{40}$ still do not recommend mpMRI prior to an initial set of biopsies. In this study, leading with mpMRI allowed $24(21.6 \%)$ men to avoid a biopsy entirely. However, the majority would remain on PSA surveillance due to the small-but understood-risk of a false-negative mpMRI. There is perhaps a concern that in less-experienced centres, overcall of images as PIRADS 3 is an issue that will expose men to unnecessary biopsies, thus reducing the benefit of an image-guided pathway. However, as the PIRADS V.2 $2^{41}$ scoring system is increasingly adopted, with its ability to define a PIRADS 4 lesion over a 3 by utilisation of the second parameter (Dynamic contrast enhanced (DCE) and Diffusion-weighted imaging (DWI) for peripheral zone and transition zone lesions, respectively), alongside its more easily understood and applicable design, should reduce such an effect going forward.

Clearly, there is enough evidence now to introduce an image-guided biopsy to the prostate cancer diagnostic pathway, bringing it in line with the current practice in other solid organ malignancies. However, currently there is concern that targeted biopsies alone risk missing areas of significant disease that appear normal on mpMRI. This may be viewed as a limitation. However, our current approach to this cohort of men was introduced after our paired analyses of mpMRI versus template biopsies demonstrated that mpMRI cognitive biopsies had equivalent detection rates to zonal mapping biopsies. ${ }^{37}$ Furthermore, numerous centres have now reported improved cancer detection rates of MRTB strategies when compared with systematic approaches, ${ }^{642}$ as well as improved biopsy efficiency and reduced false-negative rates for significant cancer. ${ }^{8}$ To underline this, another series of men who underwent both fusion MRTB and systematic TPM showed a difference of clinically significant cancer-detection rates of $4 \%$ (28\% for MRTB and $24 \%$ for systematic biopsy), although combined biopsies outperformed each approach in isolation. ${ }^{43}$ Naturally, such results have been reported by specialist centres and as such, concern remains with regard to the level of operator dependency with targeted biopsy techniques. However, authors have found no difference between cancer detection rates with targeted techniques regardless of the experience of the operator, although with TRUSGB. ${ }^{44}$ Of course, advocating for a rapid uptake of such techniques in centres with no prior experience would be optimistic. Instead, we envisage a stepwise, quality-controlled uptake of transperineal approach biopsies and mpMRI reporting before adopting targeted strategies.

As with mpMRI, MRTB is not a perfect test, both can miss significant disease. However, this is an improvement on our current standard diagnostic test which is demonstrably poor. ${ }^{27-30}$ As recent studies have shown, in comparison with TRUSGB, MRTB is more likely to detect disease once a suspicious area has been identified. ${ }^{617}$ Furthermore, the recently published PRECISION randomised controlled trial clearly demonstrated the superior clinically significant cancer detection rate of MRTB and a reduced insignificant cancer detection rate when compared with systematic TRUSGB. ${ }^{18}$

A potential limitation of the MRTB technique in this study is the use of 'cognitive fusion' rather than ultrasound/mpMRI fusion or 'in-bore' targeting. However, no superiority of one technique over another has been clearly demonstrated, while 'cognitive fusion' is clearly a less costly option. ${ }^{45}$ Another potential limitation of the targeted biopsy strategy is the 'satisfaction of search' bias. Essentially, this means that after the primary lesion is scored, less attention to detail is given to subsequent lesions which may therefore be undercalled or undersampled. However, in this series this occurred twice, only once where the secondary lesion was attributed a lower score than the primary, and in no cases did this change the proposed management. Further, in the vast majority of centres where radical treatments-rather than focal-remain the standard of care, there would likely be no change in the approach to curative therapy, save for 
planning for prostatectomy in the case of nerve-sparing procedures.

The cost of mpMRI has been cited as a reason for persisting with TRUSGB-led diagnostic pathways, ${ }^{46}$ using it instead for a second investigation in the case of a negative biopsy in a patient in whom suspicion of cancer remains. While mpMRI is indeed useful in this scenario, recent cost-effectiveness analyses have shown the long-term cost-benefits of mpMRI-led pathways when various outcomes are accounted for ${ }^{14} 4748$ due to a reduction in overdiagnosis and higher detection rates of clinically significant disease at primary biopsy. In particular, the cost analysis of the PROMIS trial cohort demonstrated that MpMRI first followed by two MRTBs detects more cancer per pound spent than a TRUSfirst biopsy strategy. ${ }^{48}$

A major advantage of our pathway is the low time to diagnosis and treatment. At a median of 8 and 20 days, respectively, the time a patient waits is significantly below the 31-day and 62-day targets set by the UK National Health Service. The meeting of these targets is a persistent challenge nationally. ${ }^{49}$ Moreover, performing an mpMRI prior to primary biopsy negates the risk of an initial false-negative biopsy significantly delaying a subsequent mpMRI due to postbiopsy haemorrhage within the prostate. This makes it difficult to localise cancer or accurately determine its size or border ${ }^{50}$ In such circumstances, the delay in diagnosis can be up to 8 weeks.

\section{CONCLUSIONS}

This novel pathway offers an alternative to standard prostate cancer diagnostic services. Attendance and cancer detection rates are high. The use of an mpMRI led pathway allows for a significant proportion of men to avoid a biopsy and for those who do, the time to diagnosis and definitive treatment is kept particularly low. The integration of both mpMRI and MRTB in the prostate cancer diagnostic pathway has shown cost-effectiveness in the long term. This is especially true where rapid diagnostics are mandated or desirable. Furthermore, today, where septic complications are of grave concern, the transperineal route is particularly advantageous. This pilot study demonstrates that similar services can be provided in appropriate centres and may be valuable to patients with a potential diagnosis of prostate cancer.

\section{Author affiliations}

${ }^{1}$ Division of Surgery and Interventional Science, University College London, London, UK ${ }^{2}$ Department of Urology, University College London Hospitals NHS Foundation Trust, London, UK

${ }^{3}$ Department of Histopathology, University College London Hospitals NHS Foundation Trust, London, UK

${ }^{4}$ Department of Radiology, University College London Hospitals NHS Foundation

Trust, London, UK

${ }^{5}$ Division of Medicine, Centre for Medical Imaging, University College London, London, UK

${ }^{6}$ Division of Surgery, Department of Surgery and Cancer, Faculty of Medicine, Imperial College London, London, UK

${ }^{7}$ Imperial Urology, Charing Cross Hospital, Imperial College Healthcare NHS Trust, London, UK

Contributors EJB drafted the manuscript and approved the final version. AF contributed to the conception of the work presented, revised the manuscript critically and approved the final version. CJ contributed to the conception of the work presented and revised the manuscript critically and approved the final version. SP contributed to the conception of the work presented and revised the manuscript critically and approved the final version. CMM contributed to the conception of the work presented and revised the manuscript critically and approved the final version. MA revised the manuscript critically and approved the final version. ME contributed to the conception of the work presented and revised the manuscript critically and approved the final version. HUA contributed to the conception of the work presented and revised the manuscript critically and approved the final version. All authors are accountable for all aspects of the work in terms of accuracy and integrity.

Funding The authors have not declared a specific grant for this research from any funding agency in the public, commercial or not-for-profit sectors.

Disclaimer The views expressed in this publication are those of the author(s) and not necessarily those of the NHS, the National Institute for Health Research or the Department of Health.

Competing interests HUA's research is supported by core funding from the UK's National Institute of Health Research (NIHR) Imperial Biomedical Research Centre. This paper is independent research funded by the National Institute for Health Research (NIHR) Imperial Biomedical Research Centre (BRC). HUA currently receives funding from the Wellcome Trust, Prostate Cancer UK, Sonacare Inc, Trod Medical and Sophiris Biocorp for trials in prostate cancer. HUA is a paid medical consultant for Sophiris Biocorp for trials work. ME's research is supported by core funding from the UK's National Institute of Health Research (NIHR) UCLH/UCL Biomedical Research Centre. He was awarded NIHR Senior Investigator in 2015. ME receives funding from NIHR-i4i, MRC, Sonacare Inc, Trod Medical, Cancer Vaccine Institute and Sophiris Biocorp for trials in prostate cancer. ME is a medical consultant to Sonacare Inc, Sophiris Biocorp, Steba Biotech, Exact Imaging and Profound Medical. CMM receives funding from the National Institute for Health Research, The European Association of Urology Research Foundation, Prostate Cancer UK, Movember and the Cancer Vaccine Institute, for clinical prostate cancer research. She has received advisory board fees for Genomic Health. HUA, ME, and CMM are all proctors for HIFU and are paid for training other surgeons in this procedure. ME and AF have loan notes/stock options in Nuada Medical Ltd (UK).

Patient consent Obtained.

Ethics approval Local ethical approval was attained through the Hospital Trust's audit commitee.

Provenance and peer review Not commissioned; externally peer reviewed.

Data sharing statement We declare there are no unpublished data from etc study.

Open access This is an open access article distributed in accordance with the Creative Commons Attribution Non Commercial (CC BY-NC 4.0) license, which permits others to distribute, remix, adapt, build upon this work non-commercially, and license their derivative works on different terms, provided the original work is properly cited, appropriate credit is given, any changes made indicated, and the use is non-commercial. See: http://creativecommons.org/licenses/by-nc/4.0/.

\section{REFERENCES}

1. Wilt TJ, Brawer MK, Jones KM, et al. Radical prostatectomy versus observation for localized prostate cancer. N Engl J Med 2012;367:203-13.

2. Klotz L, Vesprini D, Sethukavalan P, et al. Long-term follow-up of a large active surveillance cohort of patients with prostate cancer. $J$ Clin Oncol 2015;33:272-7.

3. Loeb S, Bjurlin MA, Nicholson J, et al. Overdiagnosis and overtreatment of prostate cancer. Eur Urol 2014;65:1046-55.

4. Puech P, Rouvière O, Renard-Penna R, et al. Prostate cancer diagnosis: multiparametric MR-targeted biopsy with cognitive and transrectal US-MR fusion guidance versus systematic biopsy-prospective multicenter study. Radiology 2013;268:461-.

5. Brocken P, Prins JB, Dekhuijzen PN, et al. The faster the better? -A systematic review on distress in the diagnostic phase of suspected cancer, and the influence of rapid diagnostic pathways. Psychooncology 2012;21:1-10.

6. Moore CM, Robertson NL, Arsanious N, et al. Image-guided prostate biopsy using magnetic resonance imaging-derived targets: a systematic review. Eur Urol 2013;63:125-40.

7. Siddiqui MM, Rais-Bahrami S, Turkbey B, et al. Comparison of MR/ ultrasound fusion-guided biopsy with ultrasound-guided biopsy for the diagnosis of prostate cancer. JAMA 2015;313:390-7. 
8. Schoots IG, Roobol MJ, Nieboer D, et al. Magnetic resonance imaging-targeted biopsy may enhance the diagnostic accuracy of significant prostate cancer detection compared to standard transrectal ultrasound-guided biopsy: a systematic review and metaanalysis. Eur Urol 2015;68:438-50.

9. Puech P, Potiron E, Lemaitre L, et al. Dynamic contrast-enhancedmagnetic resonance imaging evaluation of intraprostatic prostate cancer: correlation with radical prostatectomy specimens. Urology 2009;74:1094-9.

10. Hambrock T, Hoeks C, Hulsbergen-van de Kaa C, et al. Prospective assessment of prostate cancer aggressiveness using 3-T diffusionweighted magnetic resonance imaging-guided biopsies versus a systematic 10-core transrectal ultrasound prostate biopsy cohort. Eur Urol 2012;61:177-84.

11. Moore CM, Kasivisvanathan V, Eggener S, et al. START Consortium. Standards of reporting for MRI-targeted biopsy studies (START) of the prostate: recommendations from an International Working Group. Eur Urol 2013;64:544.

12. Dickinson L, Ahmed HU, Allen C, et al. Magnetic resonance imaging for the detection, localisation, and characterisation of prostate cancer: recommendations from a European consensus meeting. Eur Urol 2011:59:477-94.

13. Gaziev G, Wadhwa K, Barrett T, et al. Defining the learning curve for multiparametric magnetic resonance imaging (MRI) of the prostate using MRI-transrectal ultrasonography (TRUS) fusionguided transperineal prostate biopsies as a validation tool. BJU Int 2016;117:80-6.

14. de Rooij M, Crienen S, Witjes JA, et al. Cost-effectiveness of magnetic resonance (MR) imaging and MR-guided targeted biopsy versus systematic transrectal ultrasound-guided biopsy in diagnosing prostate cancer: a modelling study from a health care perspective. Eur Urol 2014;66:430-6.

15. Venderink W, van Luijtelaar A, Bomers JG, et al. Results of Targeted Biopsy in Men with Magnetic Resonance Imaging Lesions Classified Equivocal, Likely or Highly Likely to Be Clinically Significant Prostate Cancer. Eur Urol 2017

16. Nassiri N, Natarajan S, Margolis DJ, et al. Targeted prostate biopsy: lessons learned midst the evolution of a disruptive technology. Urology 2015;86:432-8.

17. Pokorny MR, de Rooij M, Duncan E, et al. Prospective study of diagnostic accuracy comparing prostate cancer detection by transrectal ultrasound-guided biopsy versus magnetic resonance (MR) imaging with subsequent MR-guided biopsy in men without previous prostate biopsies. Eur Urol 2014;66:22-9.

18. Kasivisvanathan V, Rannikko AS, Borghi M, et al. PRECISION Study Group Collaborators. MRI-targeted or standard biopsy for prostatecancer diagnosis. N Engl J Med 2018;378:1767-77.

19. Barentsz JO, Richenberg J, Clements R, et al. ESUR prostate MR guidelines 2012. Eur Radiol 2012;22:746-57.

20. Kasivisvanathan V, Dufour R, Moore CM, et al. Transperineal magnetic resonance image targeted prostate biopsy versus transperineal template prostate biopsy in the detection of clinically significant prostate cancer. J Urol 2013;189:860-6.

21. Fütterer JJ, Briganti $A$, De Visschere $P$, et al. Can clinically significant prostate cancer be detected with multiparametric magnetic resonance imaging? A systematic review of the literature. Eur Urol 2015;68:1045-53.

22. Dickinson L, Ahmed HU, Allen C, et al. Scoring systems used for the interpretation and reporting of multiparametric MRI for prostate cancer detection, localization, and characterization: could standardization lead to improved utilization of imaging within the diagnostic pathway? J Magn Reson Imaging 2013;37:48-58.

23. Kirkham AP, Haslam P, Keanie JY, et al. Prostate MRI: who, when, and how? Report from a UK consensus meeting. Clin Radiol 2013;68:1016-23.

24. Rosenkrantz AB, Lim RP, Haghighi M, et al. Comparison of interreader reproducibility of the prostate imaging reporting and data system and likert scales for evaluation of multiparametric prostate MRI. AJR Am J Roentgenol 2013;201:W612-8.

25. Bass EJ, Donaldson IA, Freeman A, et al. Magnetic resonance imaging targeted transperineal prostate biopsy: a local anaesthetic approach. Prostate Cancer Prostatic Dis 2017;20:311-7.

26. Ahmed HU, Hu Y, Carter T, et al. Characterizing clinically significant prostate cancer using template prostate mapping biopsy. J Urol 2011;186:458-64.

27. King CR, McNeal JE, Gill H, et al. Extended prostate biopsy scheme improves reliability of Gleason grading: implications for radiotherapy patients. Int J Radiat Oncol Biol Phys 2004:59:386-91.
28. Chen ME, Troncoso P, Johnston DA, et al. Optimization of prostate biopsy strategy using computer based analysis. $J$ Urol 1997;158:2168-75.

29. Levine MA, Ittman M, Melamed J, et al. Two consecutive sets of transrectal ultrasound guided sextant biopsies of the prostate for the detection of prostate cancer. J Urol 1998;159:471-6.

30. Meng MV, Elkin EP, DuChane J, et al. Impact of increased number of biopsies on the nature of prostate cancer identified. $J$ Urol 2006; 176:63-9.

31. Haas GP, Delongchamps NB, Jones RF, et al. Needle biopsies on autopsy prostates: sensitivity of cancer detection based on true prevalence. J Natl Cancer Inst 2007:99:1484-9.

32. Haas GP, Delongchamps N, Brawley OW, et al. The worldwide epidemiology of prostate cancer: perspectives from autopsy studies. Can J Urol 2008;15:3866-71.

33. Ghafoori M, Velayati M, Aliyari Ghasabeh M, et al. Prostate biopsy using transrectal ultrasonography; the optimal number of cores regarding cancer detection rate and complications. Iran J Radiol 2015;12:e13257.

34. Carignan A, Roussy JF, Lapointe V, et al. Increasing risk of infectious complications after transrectal ultrasound-guided prostate biopsies: time to reassess antimicrobial prophylaxis? Eur Urol 2012;62:453-9.

35. Pepdjonovic L, Tan GH, Huang S, et al. Zero hospital admissions for infection after 577 transperineal prostate biopsies using single-dose cephazolin prophylaxis. World J Urol 2017;35:1199-203.

36. Loeb S, Vellekoop A, Ahmed HU, et al. Systematic review of complications of prostate biopsy. Eur Urol 2013;64:876-92.

37. Abd-Alazeez M, Kirkham A, Ahmed HU, et al. Performance of multiparametric MRI in men at risk of prostate cancer before the first biopsy: a paired validating cohort study using template prostate mapping biopsies as the reference standard. Prostate Cancer Prostatic Dis 2014;17:40-6.

38. Ahmed HU, El-Shater Bosaily A, Brown LC, et al. PROMIS study group. Diagnostic accuracy of multi-parametric MRI and TRUS biopsy in prostate cancer (PROMIS): a paired validating confirmatory study. Lancet 2017;389:815-22.

39. Mottet N, Bellmunt J, Briers E, et al. EAU - ESTRO - SIOG Guidelines on Prostate Cancer. https://uroweb.org/wp-content/ uploads/EAU-Guidelines-Prostate-Cancer-2016.pdf (Accessed 8th Feb 2018).

40. National Institute of Clinical Excellence. Prostate cancer: diagnosis and management 2014. https://www.nice.org.uk/guidance/cg175/ chapter/1-recommendations\#assessment-2 (Accessed 8thFebruary 2018).

41. Barentsz JO, Weinreb JC, Verma S, et al. Synopsis of the PI-RADS v2 guidelines for multiparametric prostate magnetic resonance imaging and recommendations for use. Eur Urol 2016;69:41-9.

42. Valerio M, Donaldson I, Emberton M, et al. Detection of Clinically Significant Prostate Cancer Using Magnetic Resonance ImagingUltrasound Fusion Targeted Biopsy: A Systematic Review. Eur Urol 2015;68:8-19.

43. Filson CP, Natarajan S, Margolis DJ, et al. Prostate cancer detection with magnetic resonance-ultrasound fusion biopsy: The role of systematic and targeted biopsies. Cancer 2016;122:884-92.

44. Cool DW, Zhang X, Romagnoli C, et al. Evaluation of MRI-

TRUS fusion versus cognitive registration accuracy for MRItargeted, TRUS-guided prostate biopsy. AJR Am J Roentgenol 2015;204:83-91.

45. Wegelin O, van Melick HHE, Hooft L, et al. Comparing three different techniques for magnetic resonance imaging-targeted prostate biopsies: a systematic review of in-bore versus magnetic resonance imaging-transrectal ultrasound fusion versus cognitive registration. is there a preferred technique? Eur Urol 2017;71:517-31.

46. Albertsen PC, Marks LS. MRI before prostate biopsy--yes or no? $J$ Urol 2013;190:1978-80.

47. Cerantola Y, Dragomir A, Tanguay S, et al. Cost-effectiveness of multiparametric magnetic resonance imaging and targeted biopsy in diagnosing prostate cancer. Urol Oncol 2016;34:119.e1-119.e9.

48. Faria R, Soares MO, Spackman E, et al. Optimising the diagnosis of prostate cancer in the era of multiparametric magnetic resonance imaging: a cost-effectiveness analysis based on the Prostate MR Imaging Study (PROMIS). Eur Urol 2018;73:23-30.

49. England NHS. Waiting Times for Suspected and Diagnosed Cancer Patients: 2016/17 Annual Report. https://www.england. nhs.uk/statistics/wp-content/uploads/sites/2/2017/06/CancerWaiting-Times-Annual-Report-201617-1.pdf (Accessed 21st Dec 2017).

50. White S, Hricak H, Forstner R, et al. Prostate cancer: effect of postbiopsy hemorrhage on interpretation of MR images. Radiology 1995;195:385-90. 